\title{
Kinetics of relativistic runaway electrons
}

B. N. Breizman ${ }^{1}$ and P. B. Aleynikov ${ }^{2}$

1) Institute for Fusion Studies, The University of Texas, Austin, Texas, 78712 USA

2) Max-Planck-Institut fur Plasmaphysik, Greifswald, Germany

e-mail: breizman@mail.utexas.edu

\begin{abstract}
This overview covers recent developments in the theory of runaway electrons in tokamaks. Its main purpose is to outline the intuitive basis for first-principle advancements in runaway electron physics. The overview highlights the following physics aspects of the runaway evolution: (1) survival and acceleration of initially hot electrons during thermal quench, (2) effect of magnetic perturbations on runaway confinement, (3) multiplication of the runaways via knock-on collisions with the bulk electrons, (4) slow decay of the runaway current, and (5) runaway-driven micro-instabilities. The scope of the reported studies is governed by the need to understand the behavior of runaway electrons as an essential physics element of the disruption events in ITER in order to develop an effective runaway mitigation scheme.
\end{abstract}

\section{Introduction}

Runaway electrons are known to be of serious concern with regard to safe operation of large-scale tokamaks in general and ITER in particular. These electrons can multiply exponentially when the loop voltage rises above the runaway avalanche threshold and they can quickly replace a large part of the bulk electron current [1], [2]. As explained in Ref. [3] the total number of e-folds during runaway avalanche can be estimated as $I /\left(I_{A} \ln \Lambda\right)$, where $I$ is the plasma current, $\ln \Lambda$ is the Coulomb logarithm, and $I_{A}$ is the critical Alfven current,

$$
I_{A}=\frac{m c^{3}}{e}\{c g s\}=\frac{4 \pi m c}{\mu_{0} e}\{S I\} \approx 17 k A .
$$

The $I / I_{A}$ ratio is in a several hundred range for large tokamaks, which apparently makes an ITER-size tokamak susceptible to runaway avalanches, because even a modest (smaller than $\left.I /\left(I_{A} \ln \Lambda\right)\right)$ number of e-folds in a seed current may already transfer the plasma current to relativistic carriers. The characteristically large ratio of $I / I_{A}$ also indicates that the energy of the poloidal magnetic field within the plasma is greater than the total energy of the current-carrying electrons. In other words, creation of the relativistic carriers requires only a small fraction of the initial poloidal field energy. The tokamak can thus be viewed as an inductive storage device. The stored energy of the poloidal magnetic field ensures a longlasting power supply for the runaways after saturation of the avalanche. This feature separates the time-scale of the runaway production from the time-scale of the runaway current decay.

Several theoretical groups internationally are currently focused on runaway studies. The recent progress includes a first-principle description for the primary runaway electron production during the thermal quench, estimates of the runaway losses through partially destroyed magnetic flux surfaces, an improved description of fast electron collisions with heavy impurities within a Thomas-Fermi model for screening, a rigorous kinetic theory for 
relativistic runaways in the electric field that is close to the avalanche threshold, refined evaluation of the critical field for avalanche onset with a systematic description of knock-on collisions and radiative losses, demonstration of phase-space attractor that supports a peaked distribution function of the runaways, a model for current damping in a self-sustained regime of marginal criticality for the runaways, and reassessment of thresholds for the runaway-driven micro-instabilities. These new theoretical findings add substantially to the material reviewed recently in Ref. [4]. Some of them are directly relevant to current experiments on DIII-D, ASDEX-U, and JET. They also provide an important input for ITER disruption modeling and runaway mitigation strategy. Yet, we will here refrain from popular temptation to rush into conclusions and recommendations about experiments. We deliberately limit this overview to theoretical models themselves and leave it for future work to review the ongoing experiments and assess their results in a coherent way from the theory and experiment standpoints together.

\section{Critical role of bulk electron cooling}

The runaway avalanche during plasma disruption is commonly assumed to be triggered by thermal quench. Insufficient present understanding of the thermal quench mechanism is a major obstacle for complete predictive modeling of the runaway behavior. Rapid cooling of bulk electrons during thermal quench could result from fast penetration of impurities into the plasma core or from large electron heat flux to the wall along the stochastic magnetic field lines. This ambiguity needs to be resolved because it translates into large uncertainty in the bulk plasma conductivity, which makes it difficult to predict evolution of the inductive electric field. Given the lack of conclusive thermal quench theory, one may actually use the readily available electron temperature data from the present machines as an input for conceivable extrapolations to ITER. Although this semi-empirical option apparently requires additional assumptions and does not resolve fundamental physics challenges, it may still be acceptable pragmatically. From the pure theory standpoint, a possible simplified approach is to consider an extreme case in which the bulk plasma is so cold and resistive that the entire current is carried by the runaway electrons. However, the bulk electron current can actually be significant and needs to be calculated systematically rather than simply ignored. The underlying reason is that the inductive electric field causes ohmic heating of the bulk electrons, and the electrons can remain cold only in the presence of some powerful cooling mechanism not just prior to the runaway build-up but also during the build-up. The challenge is to develop a consistent theoretical model for such anomalous cooling of the bulk electrons.

Destruction of magnetic surfaces has long been identified theoretically [5] as a very powerful energy loss mechanism via electron channel when the spatial scales and the level of magnetic fluctuations are sufficient to break the surfaces globally rather than at just a few radial locations with the resonant rational values of the safety factor. Simulations of existing experiments with nonlinear MHD codes NIMROD [6], [7] and JOREK [8] show that initially unstable long wavelength modes can eventually randomize magnetic surfaces 
within a large part of the plasma volume or even globally. The magnetic field then looses its perfectly nested structure due to presumably random radial walk of every field line.

Consequently, the electrons can carry a large radial heat flux in their fast motion along the perturbed field. The magnetic fluctuations of interest tend to be nearly static on the electron transit time-scale, and the resulting heat diffusion coefficient should then be proportional to the electron thermal speed and scale as $(\delta B)^{2}$ with the perturbation level. There has been an extensive work aimed at modeling and deeper understanding of the field stochastization and its consequences [9], [10], [11], [12], [13], [14]. One of the noteworthy outcomes is that the contribution of the very short wavelengths perturbations to transport is relatively small as a result of averaging along the guiding center orbit or full gyro-orbit. This is especially relevant to higher energy particles (and hence to runaways) that have larger Larmor radii and larger orbit excursions from the equilibrium flux surfaces. Their diffusion coefficient should therefore decrease with energy. The rollover in the energy dependence of the diffusion coefficient is apparently linked to characteristic spatial scale of the perturbations. Depending on input parameters (such as spatial mode choice and mode amplitudes), one finds either global stochasticity or coexistence of stochastic areas and magnetic islands within the plasma, which is instructive for understanding experimental data, but still not sufficient for conclusive first principle predictions of electron heat transport or radial losses of the runaway electrons even when the codes simulate saturated regimes of plasma instabilities rather than just assess sensitivity to pre-specified perturbations. Also, the experimental studies are not yet ready to tell conclusively whether magnetic perturbations in the plasma core should be viewed as the dominant mechanism of electron heat transport and thermal quench [15]. The big outstanding question is whether the core or the edge controls the overall electron heat flux as well as subsequent confinement of the runaway electrons. As for the core itself, the test particle studies of stochastic transport still leave a difficult open question of how to describe the tearing-type magnetic perturbations and the resulting electron kinetics self-consistently [16]. The co-existence of destroyed magnetic surfaces and magnetic islands and the plausible significance of runaway electrons for the island dynamics is a part of this problem [17].

The impurity-based cooling mechanism appears to be more controllable experimentally than the stochastic transport. It is now the prevailing candidate for disruption mitigation via massive gas injection or pellet injection, although controllable delivery of impurities is still a challenging issue [18], [19]. The main concern about massive gas injection is that ionization of the gas at the plasma edge deters rapid gas delivery to the plasma core. This tends to change the initially favorable plasma current profile to an unstable one. Pellet injection or shattered pellet injection offer a shorter penetration time, but their implementation still requires better understanding of the ablation process and of the potentially harmful lack of toroidal symmetry in the resulting density profile. Equilibration of the impurities within the magnetic surface may require a substantial time in the case of localized injection (as seen, for example, from NIMROD simulations [20]). This aspect is essential for realistic modeling of the thermal quench. A uniform density model will underestimate the line radiation and thereby the electron cooling rate during density equilibration. Also, ambipolar expansion of 
the impurity cloud along the magnetic field can act as a cooling mechanism for the electrons with their energy being transferred to the ions.

\section{Collisions and radiation}

\subsection{Scattering rate}

Scattering of the fast electrons in fully ionized plasmas with high- $Z$ ions is known to be predominantly due to electron-ion collisions, because of the large factor $Z^{2}$ in the Coulomb collision cross section. This suggests that heavy impurities can play the dominant role in fast electron scattering even when such impurities are neutral or only partially ionized as they are in post-thermal quench plasmas. Calculation of the corresponding scattering rate needs to take into account partial shielding of the impurity nuclei by the bound electrons. This quantum-mechanical problem has recently been solved in Ref. [21] based on the Thomas-Fermi model for impurity ions, which confirms the importance of the high-Z factor and provides a more systematic and accurate derivation of the collision frequency than the previous estimates in Ref. [22]. The effect of the bound electrons on elastic collisions can actually be reasonably characterized by a potential that represents the Coulomb potential of the nucleus with an account of electron shielding:

$$
\Phi=|e| \frac{Z_{0}-Z}{r} \exp \left(-r / r_{1}\right)+|e| \frac{Z}{r} \exp \left(-r / r_{2}\right)
$$

In this expression, $Z_{0}$ is the charge of the nucleus, $Z<Z_{0}$ is the charge of the ion, and $r_{1}$ and $r_{2}$ are the shielding radii for the bound electrons and free electrons, respectively. Similar to collisions in fully ionized plasmas, the dominant contribution to the collision operator of interest comes from the small-angle collisions. To find the corresponding collision frequency $v_{e l}$, we follow a standard procedure and first calculate the scattering angle for a particular collision as

$$
\Delta \theta=\left.\frac{e}{p u} \int_{-\infty}^{\infty} u d t \frac{\partial \Phi}{\partial r}\right|_{r=\sqrt{\rho^{2}+u^{2} t^{2}}},
$$

where $\rho$ is the impact parameter and $u$ the electron velocity. We then relate $v_{e l}$ to the total rate of pitch-angle spreading of the initially cold electron beam due to collisions with various impact parameters per unit time:

$$
v_{e l}=\frac{\pi n_{a}}{2} \int(\Delta \theta)^{2} u \rho d \rho \text {. }
$$

The logarithmically diverging integral in this expression need to be truncated at the lower limit by choosing $\rho_{\min } \sim \hbar / p$. We retain only the "large logarithm" terms in Eq. (4) and take into account that $r_{1}$ is much smaller than $r_{2}$. These simplifications reduce Eq. (4) to

$$
v_{e l} \approx \frac{2 \pi n_{a} e^{4}}{p^{2} u}\left[Z^{2} \ln \left(r_{2} / r_{1}\right)+Z_{0}^{2} \ln \left(\frac{r_{1}}{2 \rho_{\min }}\right)+2\left(Z_{0}-Z\right) Z \ln 2\right] \text {. }
$$


The shielding radii $r_{1}$ and $r_{2}$ in this expression are the Thomas-Fermi radius, $r_{1}=\hbar^{2} /\left(m e^{2} Z_{0}^{1 / 3}\right)$, and the Debye radius. Expression (5) is consistent with Ref.[21], but it differs parametrically from Eq. (26) of Ref. [22] in which $\rho_{\min }$ in the large logarithm $\ln \left(r_{1} / 2 \rho_{\text {min }}\right)$ appears to be the distance where the potential energy is comparable to the kinetic energy, whereas quantum limitation $\left(\rho_{\min } \sim \hbar / p\right)$ is actually more restrictive and gives a somewhat lower collision frequency as a result. The logarithmic factor $\ln \left(r_{2} / r_{1}\right)$ in Eq. (5) is roughly the Coulomb logarithm and it is typically greater than $\ln \left(r_{1} / 2 \rho_{\text {min }}\right)$, but the large $Z_{0}^{2}$ factor can easily be decisive for how to choose the correct "effective charge" for impurity ions.

\subsection{Stopping power}

Energy losses (stopping power) of the runaway electrons are largely due to collisions with the low-energy bulk electrons. The bulk contains free electrons and bound electrons residing in partially ionized impurities. The resulting rate of energy loss can be reasonably approximated as

$$
\frac{d \mathscr{O}}{d t}=-\frac{4 \pi e^{4}}{m u}\left[n_{\text {free }} \ln \Lambda_{\text {free }}+n_{\text {bound }} \ln \Lambda_{\text {bound }}\right],
$$

where $n_{\text {free }}$ and $n_{\text {bound }}$ are the concentrations of the free and the bound electrons, and $\ln \Lambda_{\text {free }}$ and $\ln \Lambda_{\text {bound }}$ are logarithmic factors with $\ln \Lambda_{\text {free }}$ being the Coulomb logarithm whose value for relativistic electrons is roughly 18. Equation (6) combines the stopping power in fully ionized plasma with a simplified expression for the Bethe-Bloch stopping power. The logarithmic factor for the bound electrons is smaller than $\ln \Lambda_{\text {free }}$, because the characteristic cut-off impact parameter in this case is determined by the average ionization potential $I$ and is of the order of $\hbar c / I$, as opposed to $c / \omega_{p}$ for the free electrons. We thus observe that

$$
\ln \Lambda_{\text {free }}-\ln \Lambda_{\text {bound }} \approx \ln \frac{I}{\hbar \omega_{p}} .
$$

The right-hand side of Eq. (7) is typically around 9, which reconciles Eq. (6) with the following practical estimate adopted in Ref. [3]:

$$
\frac{d \mathcal{O}}{d t}=-\frac{4 \pi e^{4}}{m u} \ln \Lambda_{\text {free }}\left(n_{\text {free }}+\frac{1}{2} n_{\text {bound }}\right) \text {. }
$$

Reference [21] discusses applicability and accuracy of this expression in more detail.

\subsection{Synchrotron radiation}

If scattered in pitch-angle $\theta$, relativistic runaway electrons can lose considerable energy via synchrotron emission. In the absence of an external magnetic field, the electric field can accelerate runaway electrons until they reach the pair-production energy range, but in 
magnetically confined plasmas the runaway energies are limited, rather, by synchrotron losses that accompany pitch-angle scattering. This energy loss mechanism is particularly significant in the presence of high-Z impurities and it tends to be more important than bremsstrahlung for the ITER-relevant magnetic fields and densities [23]. Besides, this mechanism is more pertinent to large devices like ITER than to smaller machines in which radial transport may exceed synchrotron losses. Plasma is optically thin with respect to the synchrotron emission, so that the rate of energy loss per particle is [24]

$$
\frac{d \mathcal{O}}{d t}=-\frac{2 e^{4} B^{2}}{3 m^{4} c^{7}} p^{2} c^{2} \sin ^{2} \theta
$$

Measurement of this emission is a valuable runaway diagnostic technique.

Even a small initial pitch-angle of $\sqrt{3} / \gamma$ would already allow the relativistic electron to radiate half of its energy. The corresponding stopping power is $d \mathcal{O} / d x=-2 e^{2} \omega_{c}^{2} / c^{2}$ and its ratio to the Coulomb collision stopping power is $2 \omega_{c}^{2} /\left(\omega_{p}^{2} \ln \Lambda\right)$, a quantity that is on the order of unity for ITER parameters. This estimate shows that synchrotron losses can easily exceed the Coulomb collision losses if there is an efficient pitch-angle scattering mechanism for the runaways. Such scattering can be due to (1) high-Z impurities [25] and (2) micro-instabilities driven by the runaways.

Radiation reaction from synchrotron emission enters the kinetic equation for runaway electrons as a non-Hamiltonian friction force. The corresponding term in the kinetic equation must preserve the number of particles and can therefore be written as a divergence of flux vector in momentum space. The flux is apparently proportional to the local value of the distribution function, and the two ( $p$ - and $\theta-$ ) components of this vector have to convey that the radiating particles preserve their parallel velocities and that the energy loss rate for a particle in uniform magnetic field is given by Eq. (9).

These conditions immediately determine both component of the flux and thereby the radiation reaction term in the kinetic equation [26]

$$
\frac{\partial F}{\partial t}=\frac{2 e^{4} B^{2}}{3 m^{3} c^{5}}\left[\frac{\partial}{\partial p} \frac{p \sqrt{m^{2} c^{2}+p^{2}}}{m c} \sin ^{2} \theta F+\frac{m c}{p \sin \theta} \frac{\partial}{\partial \theta} \frac{p \cos \theta \sin ^{2} \theta}{\sqrt{m^{2} c^{2}+p^{2}}} F\right]
$$

(we herein imply that the distribution function $F$ includes the $2 \pi p^{2}$ factor, so that the particle density is $\left.\int F d p \sin \theta d \theta\right)$.

\subsection{Knock-on collisions}

The anticipated avalanche-type production (exponentiation) of runaway electrons involves knock-on collisions of the existing runaways with the essentially immobile bulk plasma electrons including those bound in partially ionized impurities. In contrast to small-angle collisions, the knock-on collisions produce rather energetic secondary electrons.. This aspect makes the knock-on collisions less sensitive to electron binding energies except for the deepest bound electrons whose number is however relatively small for, say, Ar or Ne. It 
is therefore appropriate to treat all bulk electrons as free ones..The cross-section for knockon collisions is then given by the Møeller scattering formula [27] :

$$
\frac{d \sigma}{d \gamma}=\frac{2 \pi r_{e}^{2}}{\gamma_{0}^{2}-1}\left[\frac{\left(\gamma_{0}-1\right)^{2} \gamma_{0}^{2}}{(\gamma-1)^{2}\left(\gamma_{0}-\gamma\right)^{2}}-\frac{2 \gamma_{0}^{2}+2 \gamma_{0}-1}{(\gamma-1)\left(\gamma_{0}-\gamma\right)}+1\right],
$$

where $r_{e}$ is the classical electron radius and $\gamma_{0}$ and $\gamma$ are relativistic factor of the primary and the secondary electron respectively. The knock-on collisions introduce a source term in the kinetic equation for the runaway electrons and can give rise to an avalanche when the after-collision energies of both electrons fall in the runaway range. This source term is apparently proportional to the total number of the target electrons (free and bound). In Ref. [3], a simplified expression for the source (in the limit of $\gamma_{0} \rightarrow \infty$ ) was used to calculate the avalanche growth rate. This approximation is justified when the driving electric field is much greater than the avalanche onset threshold [28] but it does not apply to the nearthreshold case that requires more accurate description of the source [29]. The results of Monte Carlo modeling of the avalanche with a generalized knock-on source have been reported in Ref. [30]. Numerical implementation of such source within a continuous formalism is currently in progress [31].

\section{Timeline and stages of runaway evolution}

\subsection{Anticipated sequence of events}

Guided by ITER specifications and disruption phenomenology in prior experiments, we consider a reference scenario in which the plasma current, runaway electron current and the wall current evolve as shown in Fig. 1.

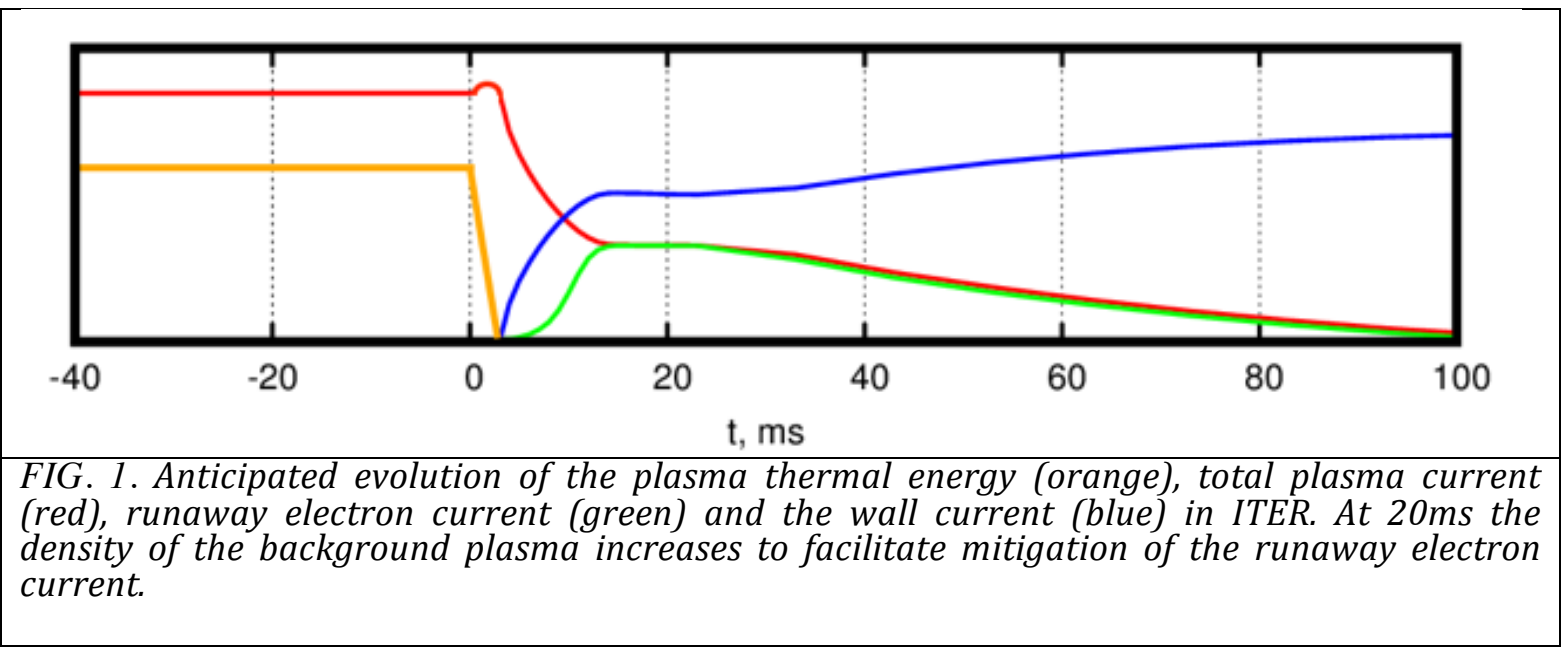

This figure presents three distinct and commonly expected phases of a disruption event in ITER: (1) primary (or "seed") runaway electron generation during thermal quench within a few (less than ten) milliseconds, (2) fast rise of the runaway population and the runaway current via avalanche mechanism with a simultaneous drop of the ohmic current within 
$10 \mathrm{~ms}$, and (3) saturation of the runaway avalanche and subsequent slow decay of the current after $10 \mathrm{~ms}$. In what follows, we overview theoretical understanding of these three phases.

\subsection{Runaway seed generation during thermal quench}

Two main mechanisms were recognized in the past as the runaway electron seed providers: (a) the diffusive leak of electrons from the maxwellian tail into the runaway regime under the influence of the driving electric field ("Dreicer generation") [32], [33], [34] and (b) survival of the "hot-tail" during thermal quench [28], [35], [36], [37], [38], [39]. The latter mechanism is most likely to prevail in ITER. This mechanism depends critically on the difference between the cooling rates of the hot-tail electrons and the bulk electrons that determine the Spitzer conductivity. The idealized picture of the hot tail scenario also implies that magnetic perturbations do not affect the lifetime of the hot electrons.

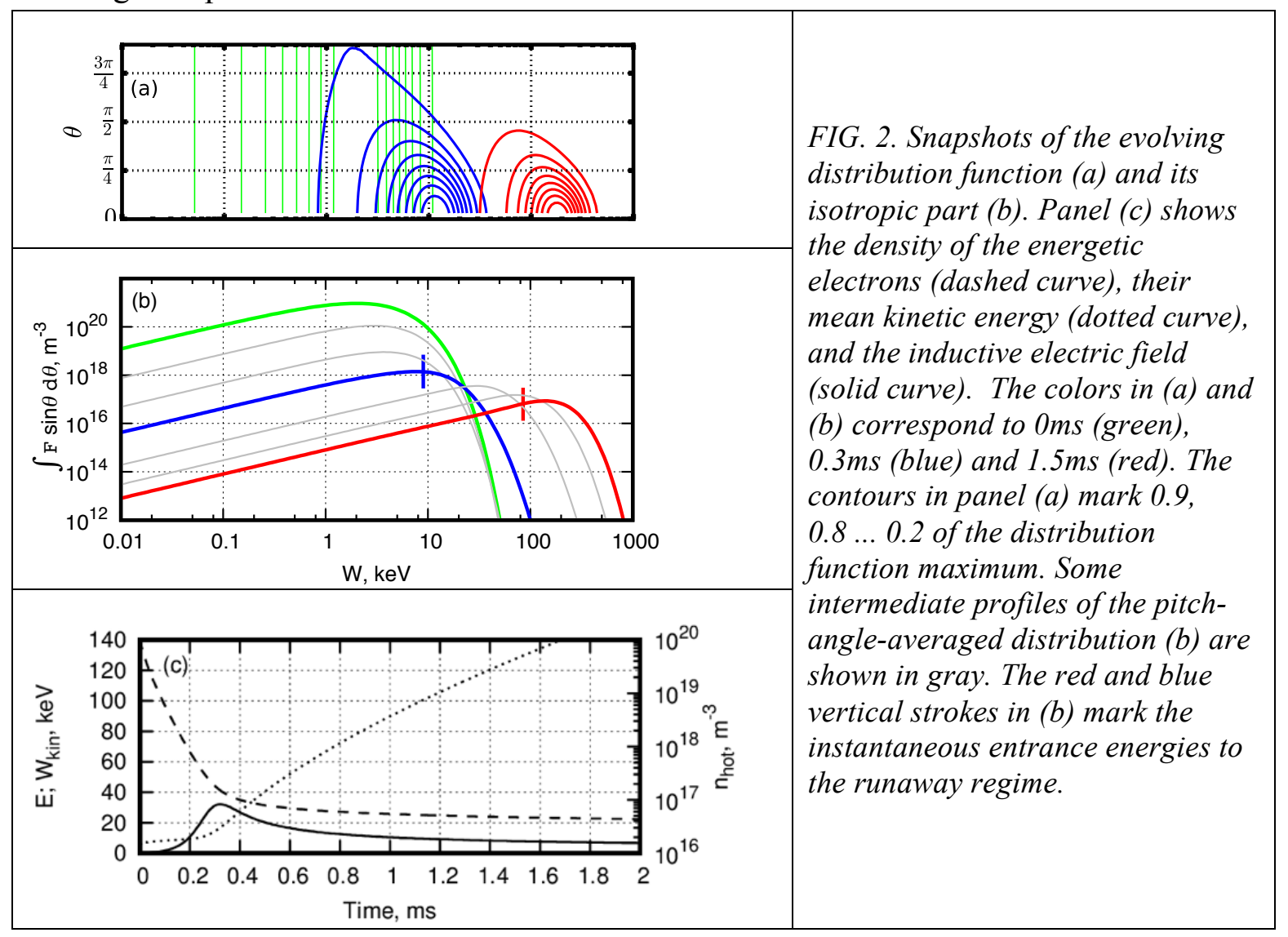

A systematic description of electron kinetics during impurity dominated thermal quench was recently developed in Ref. [40]. A 2D Fokker-Planck equation for the hot electrons and a power balance equation for the bulk plasma are solved self-consistently, with impurity radiation as the dominant energy loss mechanism. The model addresses scenario in which large amount of impurities is injected to trigger thermal quench and terminate the plasma. The behavior of the hot electron population in the limit of abundant impurities is shown in Fig. 2. The nearly isotropic initial distribution (green contours) transforms into a beam-like distribution (red contours). The electric field rises until the hot electrons reach an order-ofunity anisotropy and thereby enter the runaway regime. Due to the restrictively low conductivity of the bulk plasma, runaway electrons carry the entire pre-quench current after 
the thermal quench. This case represents a prompt conversion regime in which the runaway electron energies after the quench are in the sub-MeV range. Such runaways can be rather difficult to track with existing diagnostics.

The situation changes with the reduction of the impurity content. Figure 3 presents the runaway seed densities and energies for a range of initial plasma parameters, including those of interest for ITER.

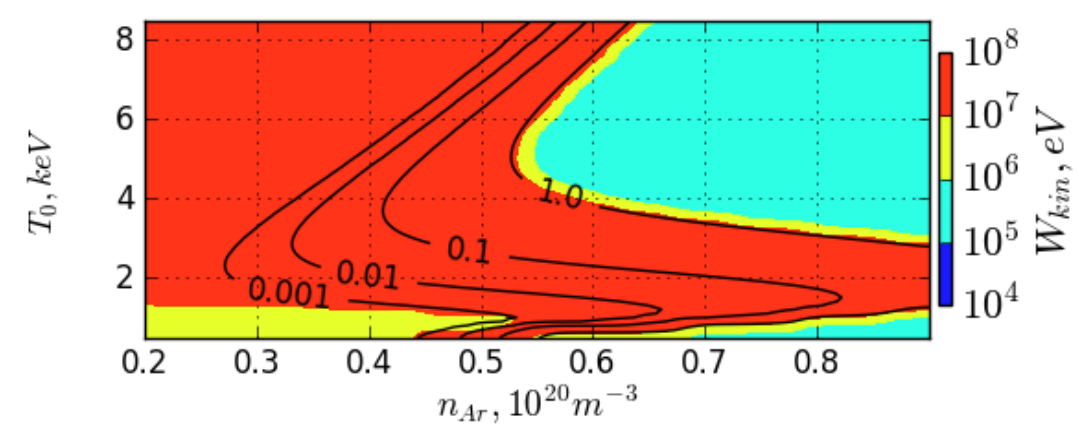

FIG. 3. Contour plot of the post-thermal-quench runaway electron density normalized to $j_{0} / e c$, where $j_{0}=1 \mathrm{MA} / \mathrm{m}^{2}$ (solid contours). Color-coded is the mean kinetic energy $W_{k i n}$ of the runaways. $T_{0}$ is the initial plasma temperature. The plasma density is $10^{20} \mathrm{~m}^{-3}$.

We find that the seed density is a non-monotonic function of the pre-quench temperature and that the seed current tends to be restrictively low in plasmas with high prequench temperatures, which is likely to cause non-monotonic runaway electron profiles in ITER and future high-temperature tokamaks. We also conclude that non-uniformity of the plasma creates a possibility for the post-quench current to be carried by two distinct runaway populations (a sub-MeV and an ultra-relativistic), which appears to be consistent with the DIII-D observations [41].

\subsection{Fast avalanche}

The presumably weak seed runaway current in ITER indicates that the post-quench electric field will be sufficiently strong to produce a rapidly growing runaway avalanche. This electric field will also characterize the resistive decay of the bulk plasma current. The avalanche growth rate found in Ref. [3] for the limiting case of very strong field leads to a simple set of circuit equations for the runaway current $I_{r e}$ and the total current $I$ within the plasma (see Ref.[42]),

$$
\frac{\partial I}{\partial s}=-\left(I-I_{r e}\right), \frac{1}{I_{r e}} \frac{\partial I_{r e}}{\partial s} \approx \frac{I_{i}}{\ln \Lambda \sqrt{Z+5}}\left(I-I_{r e}\right),
$$

where both currents are normalized to the Alfvén current, the time variable $s$ is in the units of the ohmic current decay time, and $l_{i}$ (an order of unity quantity) is the internal inductance of the plasma column. This simple model implies that the resistive decay of the wall current is negligibly slow, so that the wall is effectively a perfect conductor. 
Equations (12) have a straightforward first integral,

$$
\ln I_{r e}(\infty)+\frac{l_{i}}{\Lambda \sqrt{Z+5}} I_{r e}(\infty)=\frac{l_{i}}{\Lambda \sqrt{Z+5}} I(0)+\ln I_{r e}(0),
$$

that links the runaway current produced by the fast avalanche to the initial total current and the seed current. It is noteworthy that the dependence on the seed current is relatively week (logarithmic) and that the final runaway current is insensitive to the bulk plasma resistivity.

\subsection{Avalanche threshold and current decay}

When the fast avalanche replaces a significant part (if not the most) of the bulk electron current, the inductive electric field has to decrease considerably from its very high postthermal-quench values. This calls for special attention to the near-threshold behavior of the avalanche discussed in Ref. [29]. Besides, there are strong experimental indications [43], [44] that the threshold electric field is actually greater than the critical field needed to overcome the collisional friction for ultra-relativistic electrons.

The rates of the small-angle and knock-on collisions differ by the large Coulomb logarithm. Because of this difference, the avalanche time scale at modest electric fields is relatively slow compared to the small-angle collisional processes. This separation of time scales suggests a two-step approach to the problem. The first is to ignore the knock-on collisions and study the behavior of pre-existing runaways. The second is to use their distribution function to find the avalanche growth or damping rate. To skip the discussion of secondary geometric factors, we consider a spatially uniform plasma with a uniform magnetic field. The runaway energies in this case are limited by synchrotron radiation in partnership with pitch-angle scattering, as first shown in Ref. [45] and then emphasized in Refs. [46], [47] .The pre-existing runaways are described by the following normalized kinetic equation (see, for example, Ref. [29]):

$$
\begin{aligned}
& \frac{\partial F}{\partial t}+\frac{\partial}{\partial p}\left(E \cos \theta-1-\frac{1}{p^{2}}-\frac{p \sqrt{p^{2}+1} \sin ^{2} \theta}{\tau_{r a d}}\right) F= \\
& \frac{1}{\sin \theta} \frac{\partial}{\partial \theta} \sin \theta\left(\frac{E \sin \theta}{p} F+\frac{(Z+1)}{2} \frac{\sqrt{p^{2}+1}}{p^{3}} \frac{\partial}{\partial \theta} F+\frac{1}{\tau_{\text {rad }}} \frac{\cos \theta \sin \theta}{\sqrt{p^{2}+1}} F\right)
\end{aligned}
$$

where the particle momentum $p$ is normalized to $m c$, the electric field in normalized to the Connor-Hastie field $E_{c}$, and the times are normalized to $m c / e E_{c}$. In fully ionized plasmas, $Z$ is the ion charge, whereas in cold post-disruption plasmas $Z$ should be adjusted for impurity ions and atomic nuclei as described in Section 3.1. Also, the expression for $E_{c}$ needs to take into account collisions with the bound electrons in accordance with Section 3.2. In the case of stationary electric field, Eq. (14) has no explicit time dependence, which means that the distribution function should asymptotically achieve a steady profile with an exponentially decreasing total number of particles. This decrease is entirely due to the electron leak into the cold bulk, because a leak towards infinitely high energies is precluded by synchrotron losses. An example of the asymptotic profile is shown in Fig. 4. 


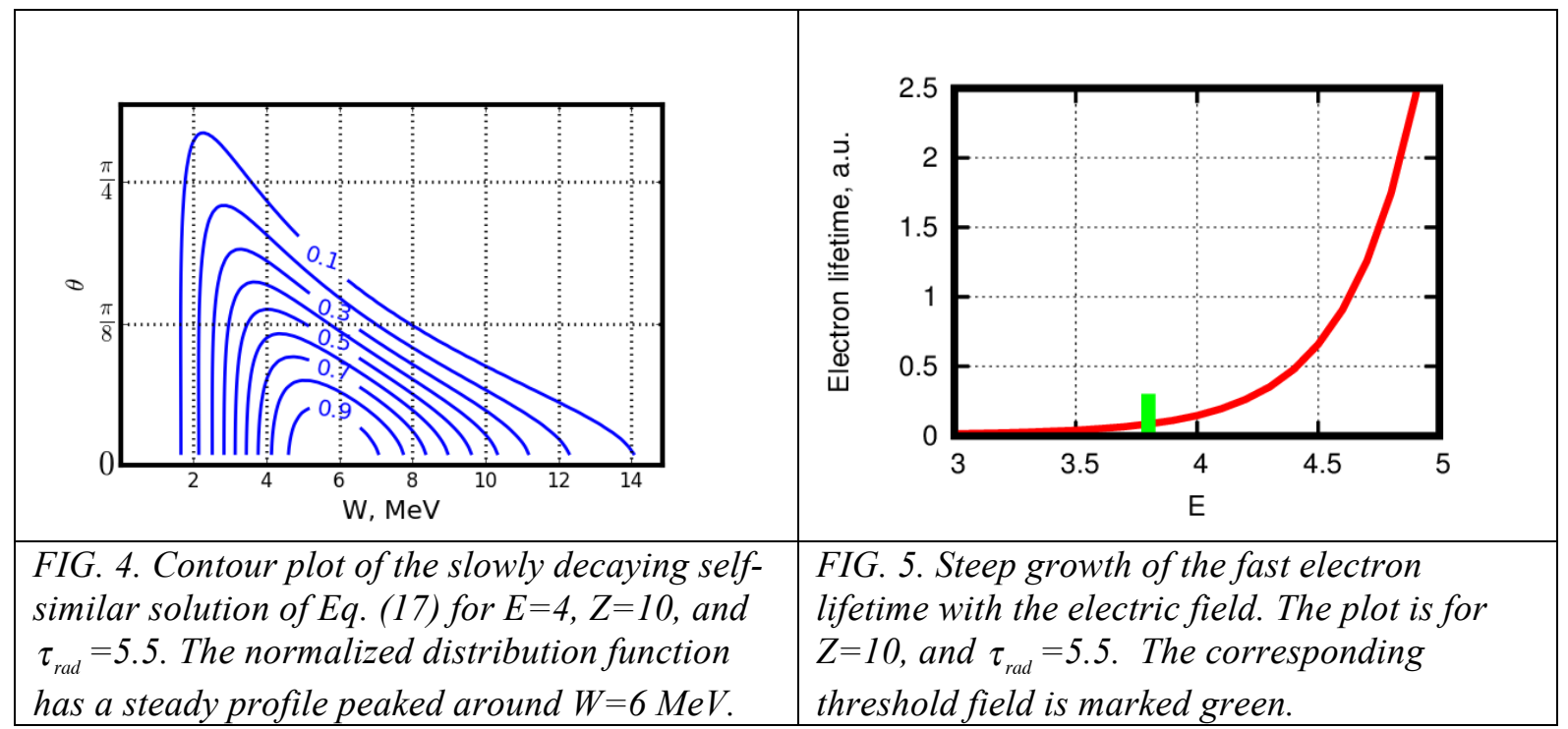

It is noteworthy that the runaways exhibit a distinctively peaked rather than monotonic energy distribution, which was reported first in Ref. [30] and then independently in Refs. [48], [49].This peaking is very likely to facilitate excitation of kinetic instabilities.

In the absence of knock-on collisions, the lifetime of the fast electron population depends on $E, Z$ and $\tau_{\text {rad }}$ as the eigenvalue of Eq. (14). This lifetime increases steeply with the electric field (see Fig. 5) when the field exceeds the threshold for runaway sustainment defined by Eq. (8) of Ref. [29] as

$$
E_{0}=1+\frac{\sqrt{2}(Z+1) / \sqrt{\tau_{r a d}}}{\sqrt[6]{1+8(Z+1)^{2} / \tau_{r a d}}}
$$

(in units of the Connor-Hastie field $E_{c}$ ). The runaway electron population can then multiply via knock-on collisions faster than the electrons leak into the bulk. Reference [29] provides a rigorous theory for the avalanche onset in the limit of negligible leak. The threshold is found to be higher and the avalanche growth rate lower than previous predictions (see Fig. 6).

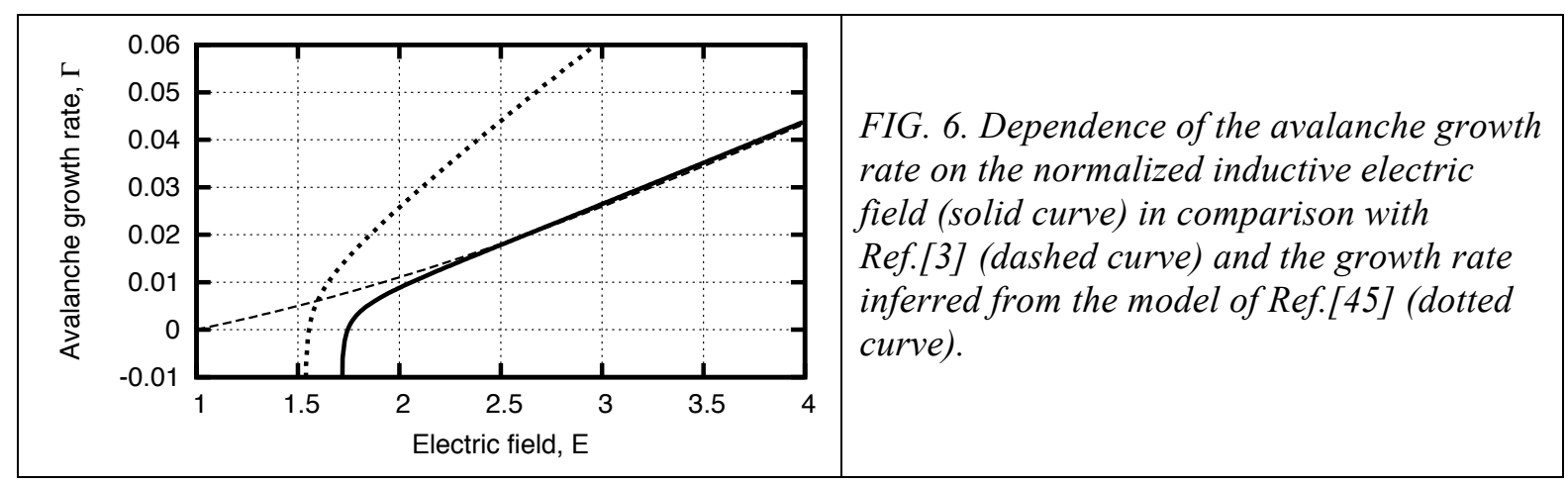

The theory also explains existence of two different threshold electric fields, and describes a mechanism for hysteresis in the runaway electron avalanche. The two different electric fields characterize a minimal field required for sustainment of the existing runaway population and a higher field required for the avalanche onset. 
The avalanche eventually saturates, and the runaway current should then decay in line with dissipation of the stored magnetic energy. This decay can be described in terms of selfsustained marginal criticality [50], which implies that the inductive electric field stays close to the avalanche threshold within the slowly evolving runaway current. Also, the runaway current density should vanish at any point where the field is subcritical, because the runaway population cannot be sustained there. These two conditions specify a nonlinear "Ohms law" for the runaway current. To illustrate the marginal criticality regime, we herein adopt Eq. (15) for the threshold field and assume that the time-scale of the avalanche growth can be estimated as

$$
\tau_{a v} \sim \ln \Lambda(m c / e) /\left(E-E_{0} E_{c}\right) .
$$

Based on dimensional arguments, the knowledge of the threshold field gives an immediate estimate for the lifetime of the runaway current $I$ [50]

$$
\tau \sim I /\left(E_{0} E_{c} c^{2}\right) .
$$

The marginal criticality scenario implies that the ratio of $\tau / \tau_{a v}$ is large for $E \sim 2 E_{0} E_{c}$, i.e.

$$
\tau / \tau_{a v} \sim e I /\left(\Lambda m c^{3}\right)>>1,
$$

which is indeed the case when the runaway current is greater than $250 \mathrm{kA}$.

Once combined with the Ampere law and the Faraday law in a nearly cylindrical configuration of the large aspect ratio torus, the marginal criticality condition gives a simple analytical model of the slowly evolving runaway current profile. This model eliminates the avalanche time-scale from the consideration and extends the analysis of Refs. [46], [51]. An example of the marginal criticality scenario is shown in Figures 7 and 8.

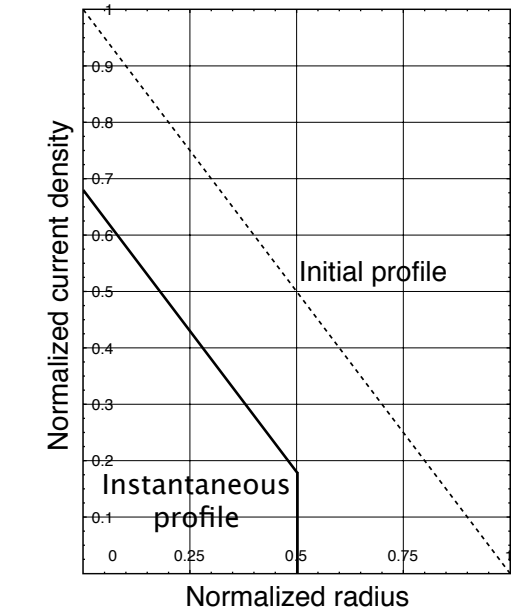

FIG. 7. Snapshot of the current profile during the decay of the runaway current. Note a sharp edge of the current-carrying channel.

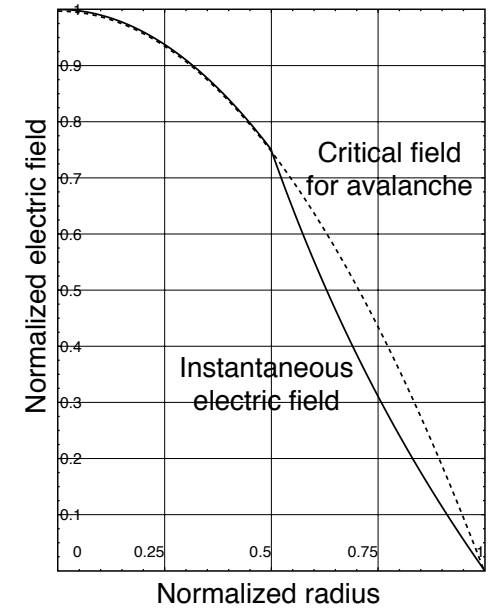

FIG. 8. Radial profile of the electric field for FIG. 7. Note that the electric field is below the critical field outside the current - carrying channel.

It should be pointed out that the presented picture of the current decay implies global equilibrium and macroscopic stability of the system whereas uncontrolled evolution of the current profile may provoke tearing instabilities. Moreover, ITER plasma is prone to vertical 
instability during disruptions [19]. The resulting vertical displacement events (VDE) can change the runaway behavior significantly [52]. Consequently, runaway kinetics and VDEs need to be modeled self-consistently. This effort is currently underway [53], [54] . Linear MHD-analysis of the post-disruption plasma in ITER has also reveals a variety of 3D MHD modes that can grow during VDEs [55]; self-consistent assessment of the runaway impact on unstable MHD modes is a yet unfulfilled challenge.

\section{Microinstabilities}

A strongly anisotropic distribution of the runaway electrons is generally prone to highfrequency kinetic instabilities that cause enhanced scattering of the runaway electrons. It is therefore important to examine plausible micro-instabilities in ITER systematically in order to first quantify the conditions for their excitation, i.e. their linear thresholds. The main potential benefit from enhanced scattering appears to be in the dramatic enhancement of energy losses via synchrotron radiation. These losses grow significantly before energetic electrons become trapped and before they diffuse to the walls. Note that the number of trapped electrons is inherently small in a large aspect ratio machine, whereas synchrotron radiation grows fast with the pitch angle for all electrons.

The relevant instabilities are in the frequency range of electron plasma waves and whistlers. In the early tokamaks, such as TM-3, T-6, TFR and others, the 'fan' instability [56], [57] was observed frequently in the presence of runaway electrons. This phenomenon has been attributed to excitation of magnetized electron plasma waves [58], [59]. The corresponding local theory (linear and quasilinear) has been instrumental in understanding the experimental data including enhanced scattering of the runaway electrons. Yet, the initial local analysis [58], [59] did not cover the effect of plasma non-uniformity on the excited waves and the system size constraints on the wave growth. Also, the analysis of magnetized plasma waves needed to be extended to other potentially unstable modes. References [60], [61], [62] reflect the efforts motivated by these unsettled issues. In particular, they highlight the role of whistler waves and suggest an estimate for their convective damping, in addition to collisional dissipation. However, the expression for the instability threshold obtained in [60], [61], [62] is problematic for two reasons. First, the collisional damping rate for whistlers was overestimated. Second, the explanation of convective damping missed the possibility of wave ducting (internal reflection) and amplification over multiple radial passes.

The characteristic wavelengths of the runaway-driven modes are typically smaller than the plasma radius, which suggests the use of the WKB approximation rather than a full wave description of the waves of interest. This approach has been implemented in a ray-tracing code COIN (convective instability) [63] that is designed to examine kinetic instabilities of a runaway beam in a tokamak for any given equilibrium configuration of the plasma and any distribution function of the runaway electrons. The code evaluates an amplification factor of the wave, $K=\int\left(\Gamma_{b}-\Gamma_{v}\right) d t$, by integrating the kinetic drive $\Gamma_{b}$ and the refined collisional damping rate $\Gamma_{v}$ along the wave packet trajectory. 


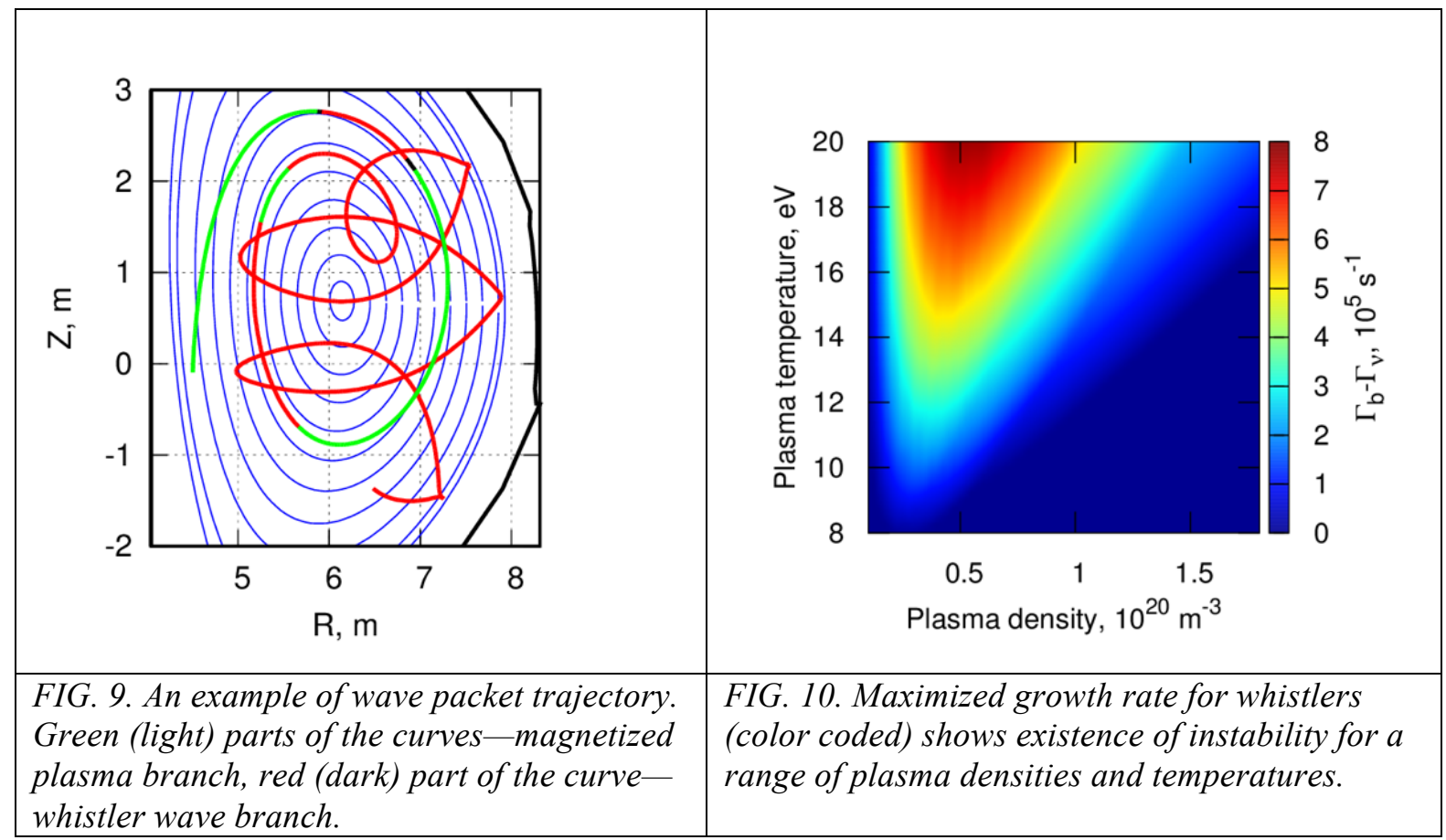

Radial non-uniformity of the tokamak plasma creates a cavity for whistlers and magnetized plasma waves with multiple transformations of these two modes into each other as shown in Fig. 7 of Ref. [63]. The calculated instability thresholds are consistent with previous experimental observations of the runaway-driven instability in several tokamaks (T-3, T-6, TFR, and T-10). The code predicts robust stability of the runaway beam in the analyzed DIIID experiments and an instability window for ITER-relevant parameters (see Fig. 10).

\section{Summary}

This overview paper is an attempt to highlight fundamental physics elements in the ongoing theoretical studies of runaway electron kinetics pertinent to large tokamaks including ITER. Listed below are recent conclusions and thoughts about next-step tasks that sum up the gist of these studies.

A combined effect of pitch-angle scattering and synchrotron radiation limits the energy gain of the runaway electrons and raises the critical field for runaway avalanche above the Connor-Hastie field.

Distribution function of the runaway electrons tends to be non-monotonic in energy with a peak at a phase-space attractor. The peaked distribution is more prone to micro-instabilities than the monotonic distribution.

Heavy impurities (irrespective of their ionization degree) are the dominant contributors to elastic scattering of the runaway electrons under plausible runaway mitigation scenarios in ITER. 
Self-consistent kinetic modeling of primary runaway formation during thermal quench shows feasibility of prompt conversion of the plasma current into a sub-MeV runaway current for heavy injection of impurities.

Non-uniformity of the plasma creates a possibility for the post-thermal-quench current to be carried by two distinct runaway populations (a sub-MeV and an ultrarelativistic).

Interaction between runaway electrons and pellets (or pellet shards) in mitigation scenarios is still an underexplored topic. The physics of pellet ablation and subsequent expansion of the ablated plasma have not yet been included into runaway modeling in a self-consistent way. A factor of particular interest is the transient non-uniformity of the ablated plasma over magnetic surface.

The present-day test particle modeling of runaway electron transport and losses needs to be extended to self-consistent description of the runaway impact on magnetic flux surface stochastization and magnetic island formation.

The time-scale of avalanche-controlled runaway current decay in ITER tends to be comparable to the time-scale of vertical displacement events (VDE), which indicates the need of self-consistent analysis of the VDEs and the runaway avalanche.

Revised thresholds for runaway-driven micro-instabilities show an instability window for whistlers in post-disruption ITER plasma with wave excitation over multiple radial passes through the plasma. This calls for nonlinear analysis of instability saturation and related assessment of the wave-induced scattering of runaway electrons. Also, possible use of the excited waves for diagnostic purposes deserves attention.

\section{Acknowledgements}

This work was supported by the U.S. Department of Energy Contract No. DEFG0204ER54742 and by ITER Contract No. ITER/CT/15/4300001178.

\section{References}

[1] Sokolov Yu.A. 1979 JETP Lett. 29218.

[2] Jayakumar R., Fleischmann H.H., and Zweben S. 1993 Phys. Lett. A 172447.

[3] Rosenbluth M.N. and Putvinski S.V. 1997 Nucl. Fusion 371355.

[4] Boozer A.H. 2015 Phys. Plasmas 22032504.

[5] Rechester A. B. and Rosenbluth M. N. 1978 Phys. Rev. Lett. 4038.

[6] Izzo V. A. 2006 Nucl. Fusion 46541.

[7] Izzo V. A. et al. 2008 Phys. Plasmas 15056109.

[8] Nardon E. et al. 2017 Plasma Phys. Control. Fusion 59014006.

[9] Yoshino R., Tokuda S. 2000 Nucl. Fusion 401293.

[10] Hauff T. and Jenko F. 2009 Phys. Plasmas 16102308. 
[11] Papp G. et al. 2012 Plasma Phys. Control. Fusion 54125008.

[12] Abdullaev S. 2014 Magnetic stochasticity in magnetically confined fusion plasmas (Cham-Heidelberg-New York-Dordrecht-London: Springer).

[13] Särkimäki K. et al. 2016 Plasma Phys. Control. Fusion 58125017.

[14] Sommariva C. et al. Simulating runaway electrons during disruptions with test particles in the JOREK code 2016 Proc. 43th EPS Conf. Plasma Phys. (Leuven, Belgium, 2016) P2.006.

[15] Zeng L. et al. 2013 Phys. Rev. Lett. 110235003.

[16] Cai H. and Fu G.. 2015 Nucl.Fusion 55022001.

[17] Boozer A.H. 2016 Phys. Plasmas 23082514.

[18] Lehnen M. et al. 2015 J. Nucl. Mater. 46339.

[19] Hollmann E. et al. 2015 Phys. Plasmas 22021802.

[20] Izzo V.A. et al. 2015 Nucl. Fusion 55073032.

[21] Zhogolev V.E., Konovalov S.V. 2014 VANT series Nucl. Fusion 3771.

[22] Mosher D. 1975 Phys. Fluids 18846.

[23] Embréus O., Stahl A., and Fülöp T. 2016 New J. Phys. 18093023.

[24] Landau L.D. and Lifshitz E.M. 1971 The classical theory of fields 3rd edn (Oxford: Pergamon Press).

[25] Aleynikova K. et al. 2013 Interaction of runaway electrons with high-Z impurities Proc. 40th EPS Conf. Plasma Phys. (Espoo, Finland 2013) O5.103.

[26] Eriksson L.-G. and Helander P. 2003 Comput. Phys. Commun. 154175.

[27] Møller C. 1932 Ann. Phys. (Berlin) 406531.

[28] Chiu S.C. et al. 1998 Nucl. Fusion 381711.

[29] Aleynikov P. and Breizman B.N., 2015 Phys. Rev. Lett. 114155001.

[30] Aleynikov P. et al. 2014 Kinetic modeling of runaway electrons and their mitigation in ITER Proc. 25th IAEA Int. Conf. on Fusion Energy (St. Petersburg 2014) TH/P338.

[31] Stahl A. et al. 2016 Nucl. Fusion 56112009.

[32] Dreicer H. 1959 Phys. Rev. 117329.

[33] Gurevich A.V. 1961 Soviet Phys. JETP 12904.

[34] Connor J.W., Hastie R.J. 1975 Nucl. Fusion 15415.

[35] Harvey R.W. et al. 2000 Phys. Plasmas 74590.

[36] Martin-Solis J.R. et al. 1975 Phys. Rev. Lett. 97165002.

[37] Helander P. et al. 2004 Phys. Plasmas 115704.

[38] Smith H. et al. 2005 Phys. Plasmas 12122505.

[39] Smith H.M. and Verwichte E. 2008 Phys. Plasmas 15072502.

[40] Aleynikov P. and Breizman B.N. 2016 Generation of runaway electrons during the thermal quench in tokamaks Proc. 26th IAEA Int. Conf. on Fusion Energy (Kyoto 2016) $\mathrm{TH} / \mathrm{P} 4-2$.

[41] Hollmann E. et al. 2015 Phys. Plasmas 22056108.

[42] Martin-Solis J.R., Loarte A., and Lehnen M. 2015 Phys. Plasmas 22082503.

[43] Martin-Solis J.R., Sanchez R., and Esposito B. 2010 Phys. Rev. Lett. 105185002.

[44] Granetz R.S. et al. 2014 Phys. Plasmas 21072506. 
[45] Martin-Solis J.R. et al. 1998 Phys. Plasmas 52370.

[46] Andersson F., Helander P., and Eriksson L.-G. 2001 Phys. Plasmas 8522.

[47] Stahl A. et al. 2015 Phys. Rev. Lett. 114115002.

[48] Hirvijoki E. et al. 2015 Journal of Plasma Physics 81475810502.

[49] Decker J. et al. 2016 Plasma Phys. Control. Fusion 58025016.

[50] Breizman B.N. 2014 Nucl. Fusion 54072002.

[51] Smith H. et al. 2006 Phys. Plasmas 13102502.

[52] Riemann J., Smith H.M., Helander P. 2012 Phys. Plasmas 19012507.

[53] Konovalov S.V. et al. 2014 Integrated modeling of ITER disruption mitigation Proc. 25th IAEA Int. Conf. on Fusion Energy (St. Petersburg 2014) TH/P3-31.

[54] Konovalov S.V. et al. 2016 Assessment of the runaway electron energy dissipation in ITER Proc. 26th IAEA Int . Conf. on Fusion Energy (Kyoto 2016) TH/7-1.

[55] Aleynikova K.O. et al. 2016 Plasma Physics Reports 42486.

[56] Alikaev V.V. et al. 1975 Sov. J. Plasma Phys. 1303.

[57] Vlasenkov V.S. et al. 1973 Nucl. Fusion 13509.

[58] Parail V.V. and Pogutse O.P. 1978 Nucl. Fusion 18303.

[59] Parail V.V. and Pogutse O.P., "Runaway electrons in a tokamak", in Reviews of Plasma Physics vol. 11, ed. M.A. Leontovich (New York: Consultans Bureau) 1986.

[60] Fülöp T. et al. 2006 Phys. Plasmas 13062506.

[61] Pokol G., Fülöp T., Lisak M. 2008 Plasma Phys. Control. Fusion 50045003.

[62] Fülöp T., Smith H., Pokol G. 2009 Phys. Plasmas 16022502.

[63] Aleynikov P. and Breizman B.N. 2015 Nucl. Fusion 55043014. 